\title{
Protein mediated membrane adhesion
}

\section{Citation}

Carlson, Andreas, and L. Mahadevan. 2015. “Protein Mediated Membrane Adhesion.” Phys.

Fluids 27 (5) (May): 051901. Portico. doi:10.1063/1.4919777.

\section{Published Version}

doi:10.1063/1.4919777

\section{Permanent link}

http://nrs.harvard.edu/urn-3:HUL.InstRepos:23600043

\section{Terms of Use}

This article was downloaded from Harvard University's DASH repository, and is made available under the terms and conditions applicable to Other Posted Material, as set forth at http:// nrs.harvard.edu/urn-3:HUL.InstRepos:dash.current.terms-of-use\#LAA

\section{Share Your Story}

The Harvard community has made this article openly available.

Please share how this access benefits you. Submit a story.

Accessibility 


\section{AIP $\left.\right|_{\text {Fyids of }} ^{\text {Phyic }}$}

\section{Protein mediated membrane adhesion}

Andreas Carlson and L. Mahadevan

Citation: Physics of Fluids 27, 051901 (2015); doi: 10.1063/1.4919777

View online: http://dx.doi.org/10.1063/1.4919777

View Table of Contents: http://scitation.aip.org/content/aip/journal/pof2/27/5?ver=pdfcov

Published by the AIP Publishing

\section{Articles you may be interested in}

Efficient elusion of viable adhesive cells from a microfluidic system by air foam

Biomicrofluidics 8, 052001 (2014); 10.1063/1.4893348

Comment on "A method to measure cellular adhesion utilizing a polymer micro-cantilever" [Appl.

Phys. Lett. 103, 123702 (2013)]

Appl. Phys. Lett. 104, 236103 (2014); 10.1063/1.4882182

Spatial anisotropy and heterogeneity in contractility and adhesion distribution may contribute to cell steering during migration

Appl. Phys. Lett. 104, 083705 (2014); 10.1063/1.4866797

Lipid membranes with transmembrane proteins in shear flow

J. Chem. Phys. 132, 025101 (2010); 10.1063/1.3285269

Cytoskeleton mediated effective elastic properties of model red blood cell membranes

J. Chem. Phys. 129, 065101 (2008); 10.1063/1.2958268

\section{Did your publisher get}

18 MILLION DOWNLOADS in 2014?

AIP Publishing did.

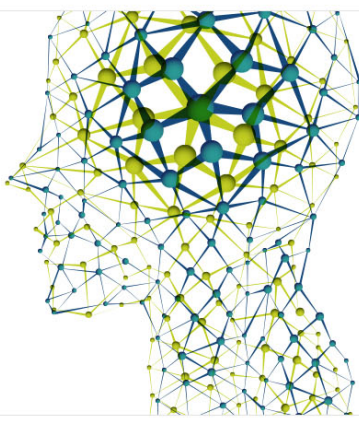




\title{
Protein mediated membrane adhesion
}

\author{
Andreas Carlson ${ }^{1}$ and L. Mahadevan ${ }^{1,2, a)}$ \\ ${ }^{1}$ School of Engineering and Applied Sciences and Wyss Institute for Biologically Inspired \\ Engineering, Harvard University, Cambridge, Massachusetts 02138, USA \\ ${ }^{2}$ Departments of Physics, and Organismic and Evolutionary Biology, Kavli Institute \\ for Bionano Science and Technology, Harvard University, Cambridge, \\ Massachusetts 02138, USA
}

(Received 30 November 2014; accepted 25 February 2015; published online 14 May 2015)

\begin{abstract}
Adhesion in the context of mechanical attachment, signaling, and movement in cellular dynamics is mediated by the kinetic interactions between membraneembedded proteins in an aqueous environment. Here, we present a minimal theoretical framework for the dynamics of membrane adhesion that accounts for the kinetics of protein binding, the elastic deformation of the membrane, and the hydrodynamics of squeeze flow in the membrane gap. We analyze the resulting equations using scaling estimates to characterize the spatiotemporal features of the adhesive patterning and corroborate them using numerical simulations. In addition to characterizing aspects of cellular dynamics, our results might also be applicable to a range of phenomena in physical chemistry and materials science where flow, deformation, and kinetics are coupled to each other in slender geometries. (C) 2015 AIP Publishing LLC. [http://dx.doi.org/10.1063/1.4919777]
\end{abstract}

\section{INTRODUCTION}

Intercellular adhesion is critical for the formation, development, and maintenance of any multicellular organism, for it allows cells to make physical contact to communicate information in both time and space. Adhesive interactions are also critically important for crawling cell movement, signaling, and recognition and enabled by the spatiotemporal patterning of the membrane embedded proteins. ${ }^{1-4}$ Previous work has focused on understanding the important biophysics of intercellular interactions using models of adhesion statics, ${ }^{5,6}$ diffusion, ${ }^{7-9}$ membrane fluctuations, and stochastic protein kinetics. ${ }^{10-16}$ We complement these approaches and describe the time and length scales associated with passive protein patterning, with a focus on the regime limited by the viscous fluid flow in the synaptic cleft. We do this by deriving a physiochemical continuum model to couple membrane deformation, protein binding and clustering, and fluid flow in the membrane gap, and to analyze it in certain prototypical settings. Our model is inspired by the transmembrane protein dynamics during cellular adhesion ${ }^{3,4}$ but formulates a class of problems that broadly links binding kinetics, hydrodynamics, and interface deformation. These coupled processes ought to be of relevance in a range of settings outside cellular dynamics in such situations as transient mechanical adhesion, physical chemistry, and problems in materials science to each other in slender geometries.

\section{MATHEMATICAL FORMULATION}

In Figure 1, we schematize cell surface-to-surface adhesion mediated by trans-membrane proteins, limiting ourselves to one-dimension to emphasize the basic physiochemical processes in the simplest geometry possible. The nucleation and growth of the protein domains are similar to

\footnotetext{
a)Electronic mail: 1m@seas.harvard.edu
} 


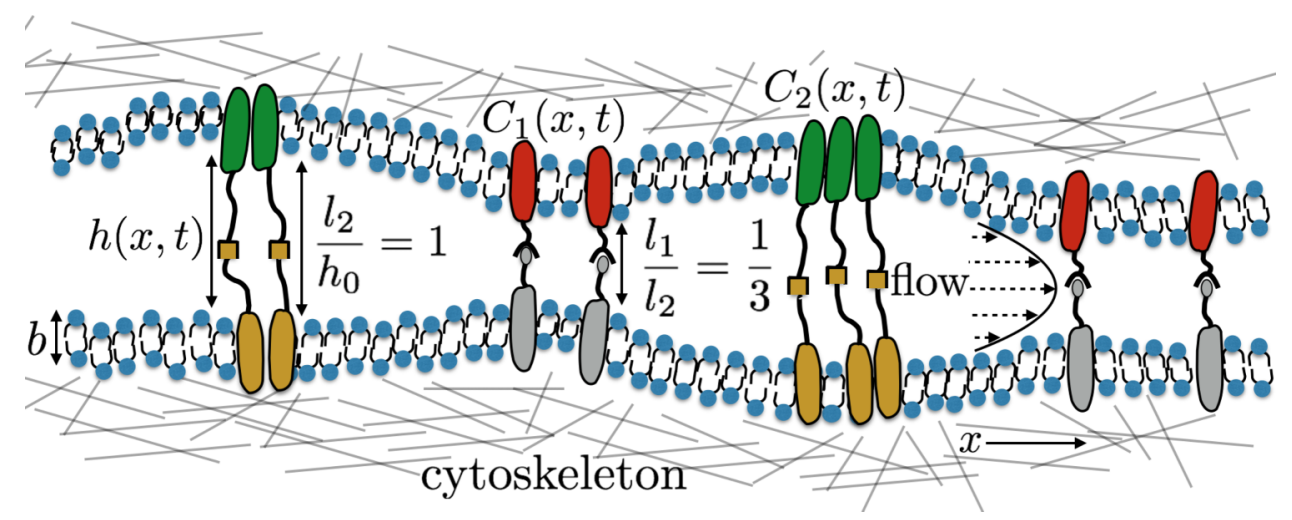

FIG. 1. A schematic of membrane adhesion mediated by proteins with two different lengths $l_{1}, l_{2}\left(l_{1} / l_{2}=15 \mathrm{~nm} / 45 \mathrm{~nm}\right.$ $=1 / 3)$ and concentrations $\left(C_{1}(x, t), C_{2}(x, t)\right)$. As the two membranes come in contact, proteins form and break bonds, which consequently generates membrane deformation and fluid flow in the gap $h(x, t) \sim O\left(l_{2} / h_{0}=1\right)$. The lateral scale $x$ spans the cell size $(\mathrm{L} \sim 10 \mu \mathrm{m})$.

that seen in two dimensions, with the main difference associated with dimensionality due to the dynamics of coarsening: protein domains can move around each other in 2D but not in 1D.

There are a variety of membrane embedded proteins which form and break bonds during the adhesion dynamics. Our specific model is inspired by the fact that the two most important adhesion proteins in the immune cell membrane ${ }^{3,4}$ have different lengths $l_{i}, i=1,2$. These two protein types have concentrations given by $C_{i}(x, t)$ that vary in space and time and their spring stiffnesses $\kappa_{i}=\kappa l_{1} / l_{i}$ that are assumed to be inversely proportional to their lengths. When these protein bonds, modeled as Hookean springs, ${ }^{17,18}$ are compressed or stretched, the force per unit area scales as $C_{i} \kappa_{i}\left(h^{*}-l_{i}\right)$ leading to a natural scaling for the pressure on the bilayer $p=\frac{p^{*}}{p_{0}}=\frac{p^{*}}{C_{0} \kappa l_{2}}$, with $C_{0}$ being the equilibrium concentration of the membrane embedded proteins per area. Here and elsewhere, we use the superscript ${ }^{*}$ to denote dimensional variables. The pressure deforms the bilayer membrane with a bending stiffness $B_{m}=\frac{E_{Y} b^{3}}{12\left(1-v^{2}\right)}$, where $E_{Y}$ is the Young's modulus, $b$ the membrane thickness, and $v$ the Poisson ratio, and transverse force balance for the membrane then yields the dimensionless equation for its height $h(x, t)$ given by

$$
p=B h_{x x x x}+C_{1}\left(h-\frac{l_{1}}{l_{2}}\right)+\frac{2 l_{1}}{l_{2}} C_{2}(h-1),
$$

where $x=L x^{*}$, with the lateral extent of the cell being $L$ and $a_{b}=\partial a / \partial b$. Scaling the membrane gap $h$ with the longest protein bond $\left(l_{2}\right) h=h^{*} / l_{2}$ leaves us with two dimensionless parameters that characterize the statics and geometry of the membrane: $B=\frac{B_{m}}{\kappa_{0} L_{0} L^{4}}$ is the ratio of membrane bending and protein deformation pressure, and $l_{1} / l_{2}=1 / 3$ is the ratio between the natural lengths of the two proteins that are of comparable length.,

The relation linking pressure, membrane deformation, and protein concentration (1) needs to be supplemented by noting that the entire process of cell adhesion occurs in an aqueous environment, with the lateral dimension $L \gg l_{2}$. The small aspect ratio $\epsilon=l_{2} / \mathrm{L} \ll 1$ of the adhesion cleft implies that the squeeze flow driven by adhesion can be very slow and is well described by an asymptotic theory for elastohydrodynamic lubrication. The thin film model couples fluid flow and the membrane deformation that has been employed in other similar situations ${ }^{19,20}$ and reads, in dimensionless form, as

$$
\frac{1}{\epsilon^{2}} h_{t}-\left(\frac{h^{3}}{12} p_{x}\right)_{x}=0
$$

where we have rescaled time here and below by the viscous time $\tau_{\mu}=\frac{\mu}{C_{0} \kappa l_{2}}$, with $\mu$ the viscosity and the pressure $p$ is given by (1). To mimic an experimental setup of an anchored lipid-bilayer that interacts with a cell, the lower surface is considered as a rigid wall. 
The dynamics of the trans-membrane proteins $\left(C_{i}=C_{i}^{*} / C_{0}\right)$, which can react, diffuse, and move by flow and are described by a dimensionless reaction-convection-diffusion equation that reads

$$
C_{i, t}=\frac{l_{1}}{l_{i}} P e^{-1} C_{i, x x}+\tau K_{i}^{o n}(h)\left(1-C_{i}\right)-\tau C_{i} \times \frac{\tau_{o n}}{\tau_{o f f}} .
$$

Here, $K_{i}(h)^{o n}$ are the Gaussian protein binding rates, which are described in detail below. By choosing $\tau_{o n} / \tau_{o f f}=1 / 3$, we favor binding to unbinding, noting that although $\tau_{o n} / \tau_{o f f}<1$ in many systems,${ }^{3,4}$ this ratio can vary substantially across biological systems. Since the membrane is much more viscous than the interstitial fluid, we neglect the influence of the advection velocity generated by the squeeze flow and the diffusion coefficient $D_{i}$ is assumed to follow the Stokes-Einstein relation, which makes $D_{i}=\left(l_{1} / l_{i}\right) D$. Although membrane diffusivity can also be determined by its anchors, our results are fairly insensitive to molecular diffusion, so that these alternate formulations lead to similar results. We see the appearance of two more dimensionless parameters, a Peclet number $P e=\frac{L^{2} C_{0} \kappa l_{2}}{D \mu}$ that describes the ratio between advection and diffusion where we note that $\frac{L C_{0} \kappa l_{2}}{\mu}$ is the viscous-spring velocity, and a dimensionless time $\tau=\frac{\tau_{\mu}}{\tau_{k}}$ that describe the ratio between the hydrodynamic time $\tau_{\mu}$ and the kinetic rate coefficient $\tau_{k}$. If $\tau>1$, bond formation is fast compared to the fluid relaxation time and the dynamics is hydrodynamically limited. Conversely when $\tau<1$, the flow relaxation time is short and the dynamics is kinetically limited.

We assume that the protein kinetics can be described minimally in terms of first order rates of binding. For diffusion limited reactions, the kinetics can be described in terms of a Kramers mean-first-passage-time over an energy barrier, ${ }^{21,22}$ determined by the equilibrium length of the molecules, and are therefore written in dimensionless form as

$$
K_{i}^{o n}(h)=\exp \left(-\left(\frac{\frac{l_{i}}{l_{2}}-h}{\frac{\sigma_{o n} l_{i}}{l_{2}}}\right)^{2}\right) .
$$

Here, we have assumed that proteins of different lengths bind at different heights with a Gaussian form for $K_{i}^{o n}$ and a scaled width $\sigma_{o n}=0.2$ relative to the most probable length $h(x, t) \sim l_{i}$. We have kept the width of the binding zone $\sigma_{o n}$ fixed in the numerical simulations. However, if $\sigma_{o n}$ is reduced protein binding would occur within a narrower range of $h$, leading to a smaller number of attached proteins in the membrane that would generate slower dynamics. Increasing $\sigma_{\text {on }}$ generates a faster dynamics with more bound transmembrane proteins, but different protein types would overlap which is unphysical. Our choice of $\sigma_{o n}=0.2$ and initial condition for $h(x, t=0) \approx 0.5$ ensure that both short and long proteins will bind. This scenario of having no "gaps" in the Gaussian distribution of protein binding implies that very little of the membrane area will be free of bound proteins. Therefore, fluctuations will be damped. Contrariwise, reducing $\sigma_{o n}$ will lead to a larger "gap" in the Gaussian distributions leading to a smaller number of bound proteins where fluctuations become more important.

We note that for hydrodynamically limited dynamics $(\tau \gg 1)$, the local protein on-rate and off-rate in (3) dominate over all gradient terms and by balancing the rate terms leads to rapid equilibration of transmembrane protein concentrations so that

$$
C_{i}^{e q}(h(x, t))=\frac{K_{i}^{o n}(h(x, t))}{K_{i}^{o n}(h(x, t))+\frac{\tau_{o n}}{\tau_{\text {off }}}},
$$

similar in shape to $K_{i}^{o n}(h)$, Figure 2(b). Later we will use (5) below to set the Dirichlet boundary conditions for $C_{i}(x=0, t)$ and $C_{i}(x=0, t)$ and to derive a simplified model for hydrodynamically limited adhesion.

Before proceeding to analyze the system, we note that including membrane tension is straightforward via the additional Laplace-like term $\sim h_{x x}$ in the expression for the pressure in Eq. (1); this sets another length scale for protein patterning. Since protein patterning occurs on nano-scales, thermal fluctuations may influence the membrane dynamics. They can be represented by a source term in (2) of the form $T_{f}\left(h^{\frac{2}{3}} N(x, t)\right)_{x},{ }^{23,24}$ where $N(x, t)$ is the spatio-temporal Gaussian white noise 


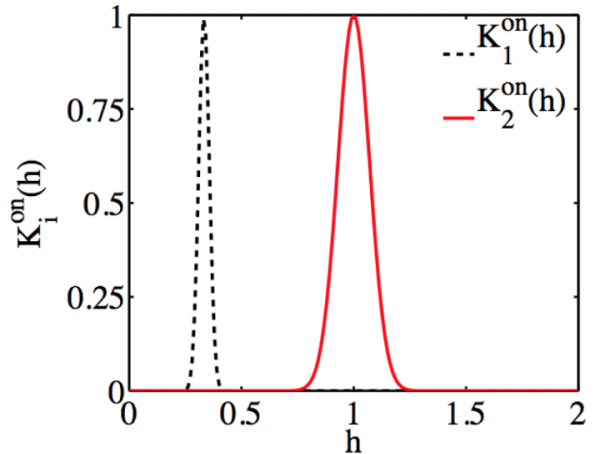

(a)

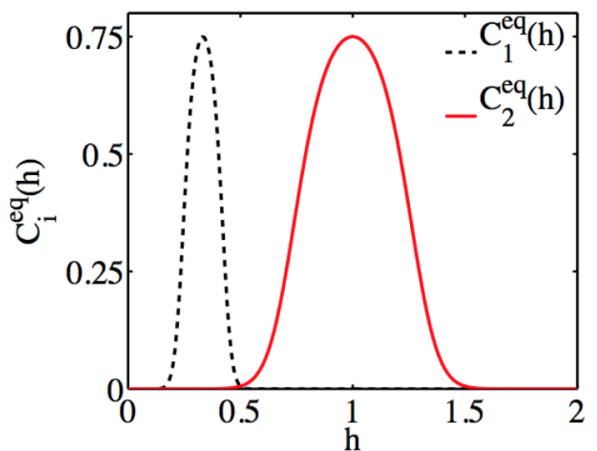

(b)

FIG. 2. (a) Graphical description of the kinetic rates of protein binding correspond to (4). The protein binding rates are a function of the membrane gap $h(x, t)$, where binding is most probable as $h(x, t) \sim l_{i}$. We use $\sigma_{o n}=0.2$ in (4) to ensure the same probability of binding for the initial condition $h(0, x) \approx 0.5$. Increasing $\sigma_{o n}$ generates a wider distribution, leading to overlapping protein species which is unphysical. Reducing $\sigma_{o n}$ makes on the other hand the region for binding narrower and would lead to a wider transition region between the two protein phases. (b) For hydrodynamically limited adhesion $\tau \gg 1$, concentration equation (3) can be reduced to (5) by assuming that the gradient terms are vanishingly small. $C_{i}^{e q}(h)$ are illustrated graphically here, where the concentrations are similar in shape to kinetic binding rates (4), but with wider distributions and shift in maxima.

with $\langle N(x, t)\rangle=0,\left\langle N(x, t) N\left(x^{\prime}, t^{\prime}\right)\right\rangle=\delta\left(x-x^{\prime}\right) \delta\left(t-t^{\prime}\right)$, and $T_{f}=\frac{k_{B} T}{\left(C_{0} h_{0}\right) L^{3}}$ is a dimensionless number representing the ratio of the thermal energy $\left(k_{B} T\right)$ and the protein spring energy $\left(\left(C_{0} \kappa h_{0}\right) L^{3}\right)$. To simplify our description of the protein patterning, we focus here on the deterministic part of the equation and let $T_{f}=0$. Linearization of (2) with respect to $\delta$ using $h=1+\delta \hat{h}$ in the absence of spring pressure yields $12 \hat{h}_{t}-\epsilon^{2} B \hat{h}_{x x x x x x}=0$, with $\hat{h}(x, t) \sim \exp (i k x+\sigma t)$ yields $\sigma \sim-B \epsilon^{2} k^{6}$, i.e., short wavelength fluctuations are strongly damped by viscosity. In contrast, long wavelength fluctuations are damped out by springs. However, if the number of transmembrane proteins is low or if they are floppy, fluctuations can be important.

To complete the formulation of the problem, we need to prescribe some initial and boundary conditions. The membrane is initialized with $h(x, t=0) \sim 0.5$ so that we have the same probability of binding both short and long proteins. This leads to an equilibrium configuration where the membrane is saturated with either short or long proteins. For boundary conditions, we assume that the membrane edge is pinned so that $h(0, t)=h(1, t)=0.5$, and further that the membrane edge is free of torques so that $h_{x x}(0, t)=h_{x x}(1, t)=0$ and maintained at constant pressure $p(0, t)=p(1, t)=0$, which allows for fluid flux into the cleft. Furthermore, for (3) and (4), we assume the equilibrium concentration at the boundary $C_{i}(0, t)=C_{i}(1, t)=C_{i}^{e q}(h(0, t))=C_{i}^{e q}(h(1, t))$, given by (5). These boundary conditions correspond to the case where a cell interacts with an anchored lipidbilayer. Alternative boundary conditions for a clamped membrane $\left(h(0, t)=h(L, t)=0.5, h_{x}(0, t)\right.$ $\left.=h_{x}(L, t)=0, p_{x}(0, t)=p_{x}(L, t)=0\right) \quad$ or $\quad$ a free edge $\quad\left(h_{x x}(0, t)=h_{x x}(L, t)=0, h_{x x x}(0, t)\right.$ $\left.=h_{x x x}(L, t)=0, p(0, t)=p(L, t)=0\right)$ that allow fluid in/out flux through the boundary do not affect the results in any significant manner. However, assuming the edge to be a wall with a pinned $\left(h_{x x}(0, t)=h_{x x}(L, t)=0\right)$ or clamped $\left(h_{x}(0, t)=h_{x}(L, t)=0\right)$ membrane $(h(0, t)=h(L, t)$ $\left.=0.5, p_{x}(0, t)=p_{x}(L, t)=0\right)$ will naturally lead to stable protein clusters at steady-state due to mass conservation.

\section{RESULTS}

The systems (1)-(4) describing the kinetics and elastohydrodynamics of membrane adhesion can be characterized in terms of the dimensionless numbers $\epsilon, P e, B$, and $\tau$ that determine the magnitude of advection, elasticity, and hydrodynamics, respectively. In the absence of a general analytical solution to the nonlinear systems (1)-(4), we use a combination of scaling analyses and numerical simulations to understand the behavior of the simulation. Our simulations used a second-order finite difference method for the spatial discretization $(\delta x=1 / 400-1 / 1600)$ and a 
time-adaptive Gear method ${ }^{25}$ for time marching. Choosing the fluid viscosity $\mu \sim 10^{-2} \mathrm{~Pa} \cdot \mathrm{s}$, protein stiffness $\kappa \sim 10^{-6} \mathrm{~N} / \mathrm{m},{ }^{7,14}$ protein diffusion coefficient $D \sim 10^{-13} \mathrm{~m} / \mathrm{s}^{2},{ }^{26,27}$ thermal energy $k_{B} T \sim 10^{-21} \mathrm{~J}$, and equilibrium membrane protein densities $C_{0} \sim 10^{14} \mathrm{~m}^{-2},{ }^{3}$ we find that the hydrodynamic time scale $\tau_{\mu}=\frac{\mu}{C_{0} l_{2}} \sim 10^{-3} \mathrm{~s}$, the bending moduli $B_{m} \sim\left(10^{-21}-10^{-18}\right) \mathrm{J}, 5,7,28$ and the inverse kinetic rate $\tau_{k} \sim 10^{-5}-10^{-1} \mathrm{~s}$. Therefore, in all our simulations we use $\epsilon=4.5 \times 10^{-3}$, $P e=5.4 \times 10^{4}$ and let $\tau \in\left[10^{-3}, 30\right]$ and $B \in\left[10^{-9}, 10^{-6}\right]$ to understand the dependence of protein patterning on the kinetics of protein binding and the scaled forces associated with membrane bending.

To derive some scaling predictions for the characteristic spatiotemporal features of intercellular adhesion, we note that the micro-cluster size $l_{c}$ is determined by balancing the scaled bending pressure $\left(B h_{x x x x}\right)$ in the membrane with the scaled protein pressure $\left(\sim C_{1}\left(h-\frac{l_{1}}{l_{2}}\right)\right)$ in $(1)$, which gives the dimensionless cluster size

$$
l_{c} \approx B^{\frac{1}{4}} \approx\left(\frac{B_{m}}{\kappa C_{0} L^{4}}\right)^{\frac{1}{4}} .
$$

In dimensional units, this reads $l_{c}^{*}=\left(\frac{B_{m}}{\kappa C_{0}}\right)^{\frac{1}{4}}$, so that for $B \in\left[10^{-9}, 10^{-6}\right]$, the scaled cluster size $l_{c} \approx 0.005-0.05$ or dimensionally $l_{c}{ }^{*} \approx\left(\frac{B_{m}}{C_{0} \kappa}\right)^{\frac{1}{4}}=50 \mathrm{~nm}-500 \mathrm{~nm}$, qualitatively consistent with experimentally observed protein domain sizes. ${ }^{3,4}$

Similarly, the characteristic time scales are associated with the time for fluid drainage through the membrane gap and are determined by the balance inherent in (2). For micro-cluster formation of size $l_{c}$, the dimensionless time scale $T_{S}$ then reads

$$
T_{S} \approx 12 \times\left(\frac{l_{c}}{\epsilon}\right)^{2} \approx \frac{12 \times B^{\frac{1}{2}}}{\epsilon^{2}}
$$

which in dimensional units is $T_{S}^{*} \approx \frac{12 \mu B_{m}^{\frac{1}{2}}}{l_{2}^{3}\left(\kappa C_{0}\right)^{\frac{3}{2}}}$. For protein patterning on the size of the cell $(L)$, the dimensionless time scale reads

$$
T_{L} \approx \frac{12}{\epsilon^{2}},
$$

which in dimensional units is $T_{L}^{*} \approx \frac{12 \mu L^{2}}{C_{0^{\kappa}} l_{2}^{3}}$. For the range of $B \in\left[10^{-9}, 10^{-6}\right]$, protein domains are predicted to form at $T_{S} \approx 25-500$, i.e., in dimensional units $T_{S} \approx 0.1 \mathrm{~s}-2 \mathrm{~s}$ and the protein pattern on the cell size to relax at $T_{L} \approx 6 \times 10^{5}$, i.e., in dimensional units $T_{L} \approx 2400 \mathrm{~s}$. Our numerical simulations below corroborate these estimates, where micro-scale protein clusters form at short times and coarsen on long length scales at much longer times as seen in Figure 3.

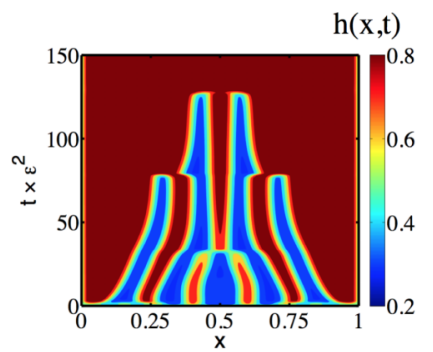

(a)

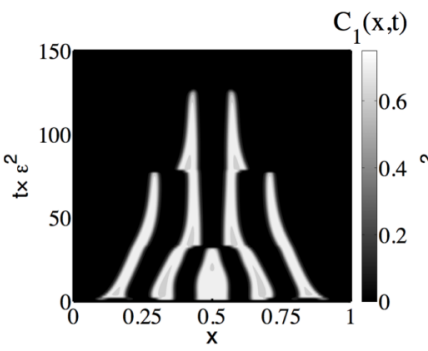

(b)

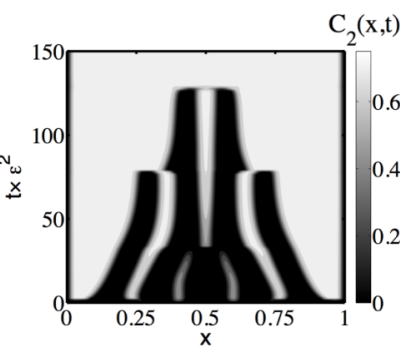

(c)

FIG. 3. Contour plots of the evolution of (a) membrane height $h(x, t)$, (b) protein concentration $C_{1}(x, t)$, and (c) protein concentration $C_{2}(x, t)$ for two protein species $i=2$ obtained by solving (1)-(4) for $B=2 \times 10^{-8}$ and $\tau=3$. The membrane shape is initialized as $h(t=0, x)=1+0.05 \times\left(\tanh ((10 x-5))^{2}-1.0\right)$, with protein concentrations corresponding to their equilibrium values $\left(C_{i}^{e q}(h(t=0, x))\right)$. At short times, protein bonds nucleate on the membrane and are sorted into small clusters. This generates membrane deformation and an inhomogeneous pressure that sets the intervening fluid in motion, causing the clusters to coarsen. 


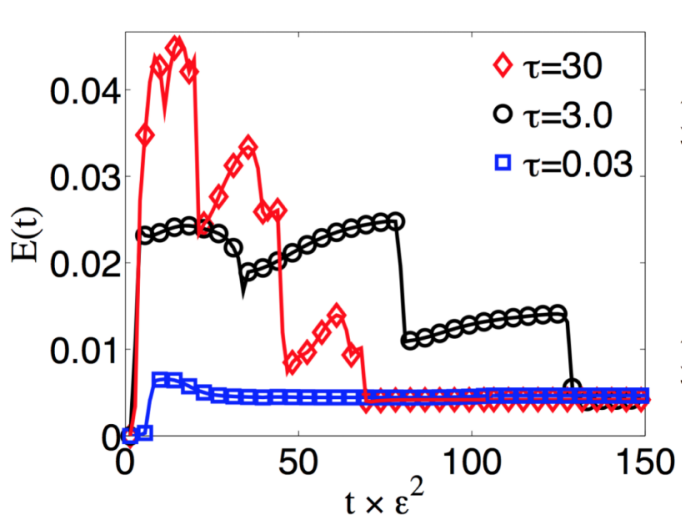

(a)
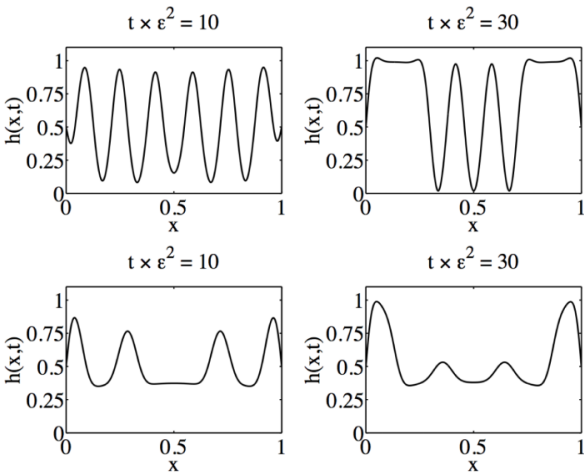

(b)

FIG. 4. (a) The coarsening dynamics of the protein patterns is illustrated by the scaled membrane bending energy $E(t)$ $=\int l_{c}^{4} \times h(x, t)_{x x}^{2} d x$ and is represented here for $B=2 \times 10^{-8}$ for three different values of $\tau=3 \times\left[10^{-2}, 1,10\right]$, corresponding to the blue filled circles, black squares, and red circles. The black squares correspond to the simulation presented in Figure 3 , linking the membrane shape and $E(t)$. In the kinetic regime $\tau>1$, the membrane deforms significantly at short times and coarsens through jumps in $E(t)$ as it slowly approaches equilibrium. In the diffusive regime $\tau<1$, the membrane is much less deformed and as $\tau$ decreases the dynamics are insensitive to variations in $\tau$. (b) $\tau=30$ and (c) $\tau=0.03$ show the different membrane shapes at two snapshots in time $t=10$ (left) and $t=30$ (right).

In Figure 3, we show the resulting numerical evolution of the spatiotemporal dynamics of the membrane height and protein concentrations by solving (1)-(4). The initial pressure minimum at the center $x=0.5$ generates a centripetal flow from the edges to the center, which transports the protein clusters and effectively coarsens the protein pattern. At equilibrium, the membrane has a nearly flat shape at the center of the membrane and the adhesion is dominated by the binding of only the long proteins. We see that the dynamics of protein patterning occurs in two stages: initially into micro-clusters at a scale $l_{c}$ over a short time $T_{S}$ and eventually at the scale of the cell $L$ over a long time $T_{L}$, corroborating our scaling predictions made in (6)-(8).

To illustrate the competition between the protein binding kinetics and the squeeze flow in the membrane gap, we plot the scaled bending energy $E(t)=\int l_{c}^{4} h_{x x}^{2} d x$ for different choices of $\tau$ in Figure 4(a). In the hydrodynamically limited regime $\tau>1$, the bending energy rapidly increases at short times $t \sim T_{s}$ due to the nucleation of protein micro-clusters before reaching its maximum. Thereafter, the membrane starts to coarsen as the clusters move towards each other by the centripetal squeeze flow and generates nearly discrete jumps in $E(t)$ as they coalesce (Figure 4(b)). In the kinetically limited regime $(\tau<1)$, there are fewer micro-clusters of larger size (Figure 4(c)), and the dynamics is diffusively dominated.

\section{DISCUSSION}

Our theory for membrane adhesion accounts for the kinetics of membrane embedded proteins, membrane deformation, and slow drainage in the narrow gap, allowing us to characterize the dynamics of the process (Fig. 4). In particular, we show that the competition between membrane deformation, fluid flow, and protein binding kinetics regulates the formation and organization of protein domains on short and long lengths and time scales. Our simple scaling estimates for the characteristic time and length scales are consistent both with observations and our detailed numerical simulations. We now turn to two simplifications that arise naturally as limiting cases of our model, before concluding with a brief discussion of broader generalizations that might be worth pursuing. In the regime of very fast binding kinetics, i.e., hydrodynamically limited dynamics $\tau \gg 1$, our model (1)-(4) can be further reduced. Similarly, when the adhesion dynamics is mediated by a single type of transmembrane proteins, i.e., $i=1$, our model also simplifies for obvious reasons. 


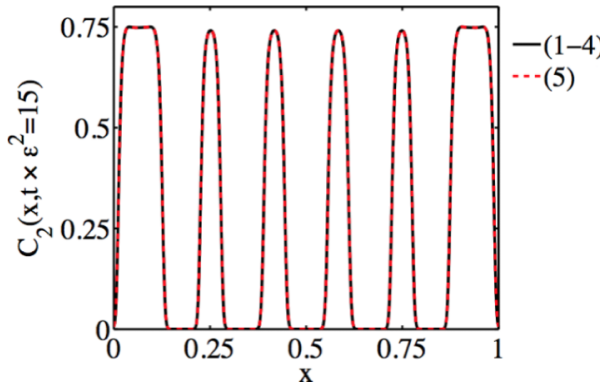

(a)

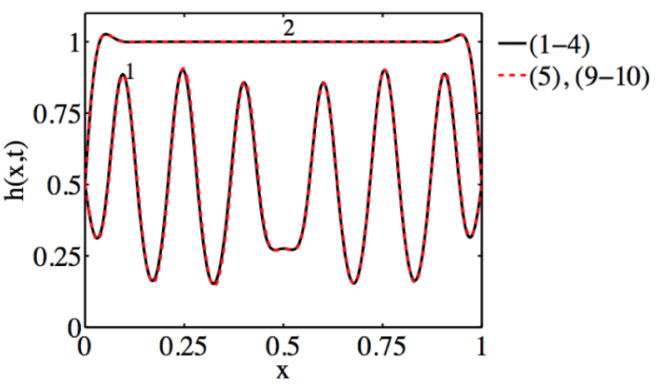

(b)

FIG. 5. Comparison of full models (1)-(4) with simplified model (5), (9), and (10) for (a) the protein concentration $C_{2}(x, t)$ and (b) $h(x, t)$ when $B=2 \times 10^{-8}$ and $\tau=30$. (a) The steady-state membrane bound protein $C_{2}\left(x, t \times \epsilon^{2}=15\right)$ is predicted by (1)-(4) and the analytical equilibrium concentration $C_{2}^{e q}\left(h\left(x, t \times \epsilon^{2}=15\right)\right)$ is predicted by (5) and agree well. $h\left(x, t \times \epsilon^{2}=15\right)$ is used as input to (5). (b) The results of full models (1)-(4) are shown using solid lines and those using reduced models (5), (9), and (10) are shown using dashed lines at two different points in time $t \times \epsilon^{2}=7,28$.

In the hydrodynamically limited regime of intercellular adhesion, clusters of membrane-bound proteins reorganize by domain coarsening similar to our observations. ${ }^{3}$ In this regime $(\tau \gg 1)$, we can reduce (3) and (4) by noting that the concentrations reach their equilibrium values quickly so that $C_{i}=C_{i}^{e q}(h(x, t))$ in (5). To verify that this is a reasonable approximation, we compare the prediction from (5) and the numerical solution of the full model (1)-(4). In Figure 5(a), we show that this is indeed the case.

Next, we turn to the pressure in the membrane gap, which we obtain by combining (1) and (5),

$$
p^{e q}=B h_{x x x x}+C_{1}^{e q}(h)\left(h-\frac{l_{1}}{l_{2}}\right)+\frac{2 l_{1}}{l_{2}} C_{2}^{e q}(h)(h-1) .
$$

At equilibrium, $p^{e q}=p^{e q}(x, t=\infty)=0$ and with (5), the equilibrium height field reduces to an ordinary differential equation, which we have verified in additional simulations but not presented here. In fact, we can go beyond the equilibrium profiles by substituting $p$ with $p^{e q}$ in (2) to derive a simplified description for the spatiotemporal evolution of membrane shape and protein patterning that is given by (5)-(9) and

$$
\frac{1}{\epsilon^{2}} h_{t}-\left(\frac{h^{3}}{12} p_{x}^{e q}\right)_{x}=0
$$

Direct comparison between the reduced mathematical model given by (5), (9), and (10) and full model (1)-(4) for the membrane shape at different points in time are shown in Figure 5(b) and

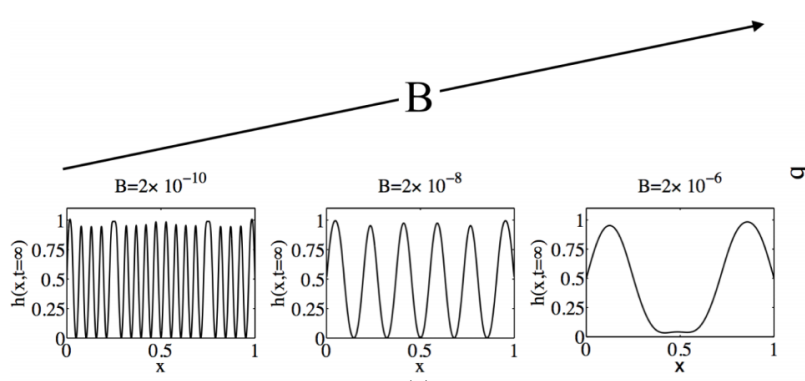

(a)

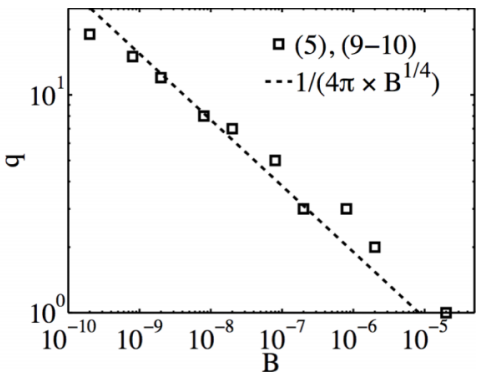

(b)

FIG. 6. (a) The three panels, starting from left to right, show the equilibrium membrane shapes as a function of $B$. These results are obtained by solving (5), (9), and (10) numerically, assuming the membrane is pinned at the boundary $\left(h_{x x}\right.$ $\left.(x=0, t)=h_{x x}(x=1, t)=0, h(x=0, t)=0.5, h(x=1, t)=0.5\right)$ and with no fluid flux $\left(p_{x}(0, t)=p_{x}(1, t)=0\right)$. (b) To further test our scaling prediction for the characteristic membrane deformation $l_{c} \approx B^{\frac{1}{4}}$, we systematically analyze the steady-state membrane pattern as a function of $B \in\left[10^{-10}, 10^{-5}\right]$. The dominant wavenumber $q$ extracted from each simulation using a fast-Fourier transform (square markers) corroborates our scaling prediction (6) with $q \approx \frac{1}{4 \pi \times l_{c}}$ (dashed line). 


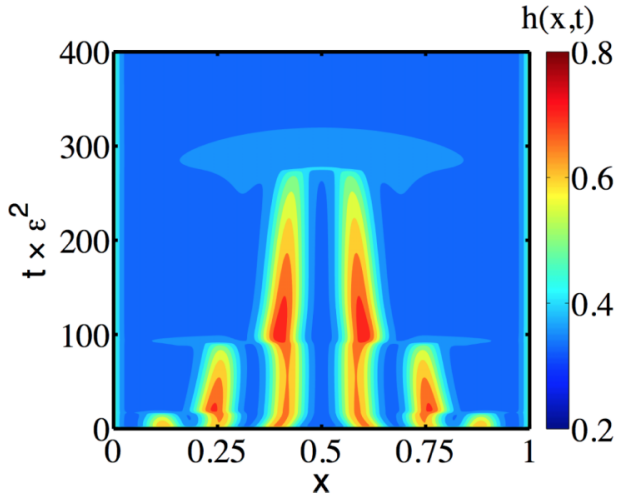

(a)

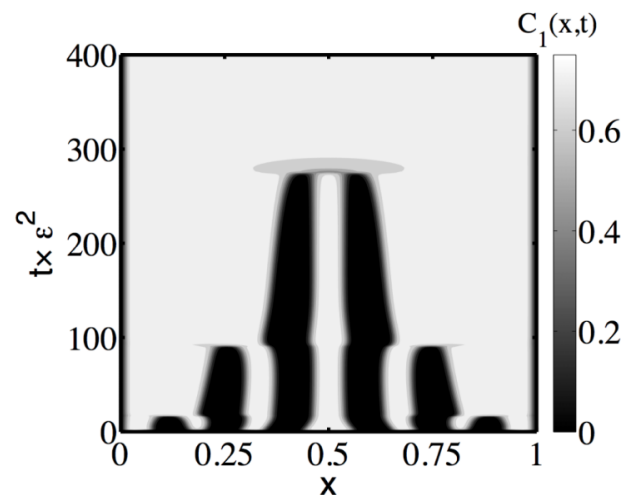

(b)

FIG. 7. Contour plots of the evolution of (a) membrane height $h(x, t)$, (b) protein concentration $C_{1}(x, t)$ for a single protein species $i=1$, obtained by solving (1)-(4) for $B=2 \times 10^{-8}$ and $\tau=3$. The membrane shape is initialized as $h(t=0, x)$ $=1+0.05 \times\left(\tanh ((10 x-5))^{2}-1.0\right)$, with protein concentrations corresponding to its equilibrium value $\left(C_{1}^{e q}(h(t=0, x))\right)$. The membrane coarsening dynamics for a single type $i=1$ of adhesion proteins is similar to the case with two protein types $i=2$ of different lengths as shown in Figure 3 .

demonstrate that the reduced model gives an accurate representation of the spatiotemporal features in this limit.

To further test our scaling prediction for the characteristic membrane deformation $l_{c}$ in (6), we simulate the equilibrium membrane shape using reduced model (5), (9), and (10) and treat the boundaries as walls with no fluid flux, i.e., $p_{x}(x=0, t)=p_{x}(x=1, t)=0$ and keeping the membrane pinned, i.e., $h_{x x}(x=0, t)=h_{x x}(x=1, t)=0, h(x=0, t)=h(x=1, t)=0.5$. The stationary membrane shape in Figure 6(a) shows that the final pattern depends on $B$. A fast-Fourier transform of the equilibrium patterns is used to determine the characteristic wavenumber $q$ and confirms our scaling $(6) q \approx \frac{1}{l_{c}}$ (Figure 6(b)).

Another simplification of our model (1)-(4) arises by considering just one type of transmembrane adhesion proteins, i.e., $i=1$. In Figure 7, we show the results of our simulation for $i=1$ and find similar membrane coarsening dynamics as for the case with two protein types $i=2$ of different lengths (Figure 3); here too scaling relations (6)-(8) remain valid. However, we note that the time scale for the coarsening is much larger when $i=1$, a consequence of a smaller driving force due to a lower number of attached proteins. In addition, the averaged film height is smaller that also generates a larger viscous resistance that slows the dynamics.

Our study has exposed a range of interesting phenomena that couple hydrodynamics to kinetics and elastic deformations in thin films, problems that have a range of applications in biology, chemistry, and materials science. Natural next steps include quantifying the influence of thermal fluctuations, understanding the interplay between membrane tension and bending, and accounting for the active dynamics of the membrane proteins linked to the cytoskeleton, while also accounting for patterns that form when two-dimensional membranes adhere to a substrate.

\section{ACKNOWLEDGMENTS}

We acknowledge funding for this research provided by the Wyss Institute and Kavli Institute for BioNano Science and Technology. The computations in this paper were run on the Odyssey cluster supported by the FAS Division of Science, Research Computing Group at Harvard University.

${ }^{1}$ R. Parthasarathy and J. T. Groves, "Protein patterns at lipid bilayer junctions,” Proc. Natl. Acad. Sci. U. S. A. 101, 12798 (2004).

2 J. T. Groves, "Bending mechanics and molecular organization in biological membranes," Annu. Rev. Phys. Chem. 58, 697 (2007).

${ }^{3}$ A. Grakoui, S. K. Bromley, C. Sumen, M. M. Davis, A. S. Shaw, P. M. Allen, and M. L. Dustin, "The immunological synapse: A molecular machine controlling T cell activation," Science 285, 221 (1999). 
${ }^{4}$ C. R. Monks, B. A. Freiberg, H. Kupfer, N. Sciaky, and A. Kupfer, "Three-dimensional segregation of supramolecular activation clusters in T cells," Nature 395, 82 (1998).

${ }^{5}$ J. F. Allard, O. Dushek, D. D. Coombs, and P. A. Merwe, "Mechanical modulation of receptor-ligand interactions at cell-cell interfaces," Biophys. J. 102, 1265 (2012).

${ }^{6}$ F. Y. Leong and K.-H. Chiam, "Adhesive dynamics of lubricated films," Phys. Rev. E 81, 04923 (2010).

${ }^{7}$ S. Y. Qi, J. T. Groves, and A. K. Chakraborty, "Synaptic pattern formation during cellular recognition," Proc. Natl. Acad. Sci. U. S. A. 98, 6548 (2001).

${ }^{8}$ N. J. Burroughs and C. Wülfing, "Differential segregation in a cell-cell contact interface: The dynamics of the immunological synapse," Biophys. J. 83, 1784 (2002).

${ }^{9}$ N. J. Burroughs and P. A. van der Merwe, "Stochasticity and spatial heterogeneity in T-cell activation," Immunol. Rev. 216, 69 (2007).

${ }^{10}$ T. R. Weikl, J. T. Groves, and R. Lipowsky, "Pattern formation during adhesion of multicomponent membranes," EPL 59, 916 (2002).

${ }^{11}$ T. R. Weikl and R. Lipowsky, "Pattern formation during T-cell adhesion,” Biophys. J. 87, 3665 (2004).

${ }^{12}$ M. T. Figge and M. Meyer-Hermann, "Modeling receptor-ligand binding kinetics in immunological synapse formation," Eur. Phys. J. D 51, 153 (2009).

${ }^{13}$ M. J. Paszek, D. Boettiger, V. M. Weaver, and D. A. Hammer, "Integrin clustering is driven by mechanical resistance from the glycocalyx and the substrate," PLoS Comput. Biol. 5, e1000604 (2009).

${ }^{14}$ E. Reister-Gottfried, T. Bihr, U. Seifert, and A.-S. Smith, "Two intertwined facets of adherent membranes: Membrane roughness and correlations between ligand-receptors bonds," New J. Phys. 13, 025003 (2011).

${ }^{15}$ E. Reister-Gottfried, K. Sengupta, B. Lorz, E. Sackmann, U. Seifert, and A.-S. Smith, "Dynamics of specific vesiclesubstrate adhesion: From local events to global dynamics," Phys. Rev. Lett. 101, 208103 (2008).

${ }^{16}$ T. Bihr, U. Seifert, and A.-S. Smith, "Nucleation of ligand-receptor domains in membrane adhesion,” Phys. Rev. Lett. 101, 258101 (2012).

${ }^{17}$ The protein stiffness can in certain instances be a function deformation/flow, ${ }^{18}$ but we use here the simplest description with a constant protein spring stiffness.

${ }^{18}$ A. Salas, M. Shimaoka, A. N. Kogan, C. Harwood, U. H. von Andrian, and T. A. Springer, "Rolling adhesion through an extended conformation of integrin $\alpha_{L} \beta_{2}$ and relation to $\alpha$ I and $\beta$ I-like domain interaction," Immunity 20, 1393 (2004),

${ }^{19}$ A. E. Hosoi and L. Mahadevan, "Peeling, healing, and bursting in a lubricated elastic sheet," Phys. Rev. Lett. 93, 137802 (2007).

${ }^{20}$ A. Oron, S. H. Davis, and S. G. Bankoff, "Long-scale evolution of thin liquid films," Rev. Mod. Phys. 69, 93 (1997).

${ }^{21}$ H. A. Kramer, "Brownian motion in a field of force and the diffusion model of chemical reactions," Physica 7(4), 284 (1940).

${ }^{22}$ G. I. Bell, M. Dembo, and P. Bongrand, "Cell adhesion. Competition between nonspecific repulsion and specific bonding," Biophys. J. 45, 1051 (1984).

${ }^{23}$ B. Davidovitch, E. Moro, and H. A. Stone, "Spreading of viscous fluid drops on a solid substrate assisted by thermal fluctuations," Phys. Rev. Lett. 95, 244505 (2005).

${ }^{24}$ G. Grün, K. Mecke, and M. Rauscher, "Thin-film flow influenced by thermal noise,” J. Stat. Phys. 122, 1261 (2006).

${ }^{25}$ C. W. Gear, "Simultaneous numerical solution of differential-algebraic equations," IEEE Trans. Circuit Theory 18, 89 (1971).

${ }^{26}$ C.-J. Hsu, W.-T. Hsieh, A. Waldman, F. Clarke, E. S. Huseby, J. K. Burkhardt, and T. Baumgart, "Ligand mobility modulates immunological synapse formation and T cell activation," PLoS One 7, e32398 (2012).

${ }^{27}$ B. Favier, N. J. Burroughs, L. Wedderburn, and S. Valitutti, "TCR dynamics on the surface of the living T cell," Int. Immunol. 13, 1525-1532 (2001).

${ }^{28}$ R. Simson, E. Wallraff, J. Faix, J. Niewöhner, and G. Gerisch, "Membrane bending modulus and adhesion energy of wild-type and mutant cells of dictyostelium lacking talin or cortexillins," Biophys. J. 74, 514 (1998). 